\title{
Finalités et valeurs de différentes politiques d'éducation à la soutenabilité
}

Yves Girault, Aurélie Zwang et Agnieszka Jeziorski

\author{
(2) OpenEdition \\ Journals \\ Édition électronique \\ URL : http://journals.openedition.org/ere/698 \\ DOI : $10.4000 /$ ere.698 \\ ISSN : 2561-2271 \\ Éditeur \\ Centr'ERE
}

Référence électronique

Yves Girault, Aurélie Zwang et Agnieszka Jeziorski, « Finalités et valeurs de différentes politiques d'éducation à la soutenabilité », Éducation relative à l'environnement [En ligne], Volume 11 | 2014, mis en ligne le 20 décembre 2013, consulté le 21 février 2020. URL : http://journals.openedition.org/ere/698 ; DOI : 10.4000/ere.698 


\title{
Finalités et valeurs de différentes politiques d'éducation à la soutenabilité
}

\author{
Yves Girault, Aurélie Zwang et Agnieszka Jeziorski
}

1 Nous souhaitons montrer dans cet article que, contrairement à une idée préconçue, la mise en place progressive de l'ESD $^{1}$ (Education à la soutenabilité) au niveau international traduit des interprétations très différentes des textes de l'ONU et, tout comme l'Éducation relative à l'environnement (ERE), cette éducation s'est construite très progressivement selon une mosaïque diversifiée de pratiques et de philosophies sous-jacentes, propres aux pays d'origine et à des contextes d'émergence particuliers qui ne cessent donc d'évoluer. Pour illustrer notre propos, nous allons présenter un éventail assez large de diverses modalités de la déclinaison de cette éducation dans des pays différents, que nous avons retenus à cet effet. Dans les lignes qui suivent, et pour bien cadrer notre propos, nous allons tout d'abord rappeler quelques grandes étapes de la mise en place de l'ESD au niveau international.

\section{L'Education for Sustainable Development au niveau international}

2 Le chapitre 36 du programme d'action des Nations Unies pour le $21^{\mathrm{e}}$ siècle, qui concerne spécifiquement l'éducation, la formation et la sensibilisation, préconise notamment de "réorienter l'éducation vers un développement durable (CNUED, 1992). En 1994, l'UNESCO lance, dans le cadre de son mandat pour appliquer ce chapitre, l'initiative internationale «Educating for a Sustainable Future: Environment, Population and Sustainable Development » (EPSD), traduit en français par «Éducation pour un Avenir Viable»(EAV). Celui-ci va insuffler le Programme international sur l'éducation, la sensibilisation du public et la formation à la viabilité en 1996, juste après la fin du Programme International d'ERE (1975-1995) (UNESCO, 2002). 
3 En préparation à la conférence internationale de Thessalonique de 1997 sur "l'environnement et la société : éducation et sensibilisation du public à la viabilité ", un document stratégique trace les premières grandes lignes de l'EAV, comme un «premier effort pour articuler les messages fondamentaux de l'éducation pour un développement durable » (UNESCO, 1997, p. 5). Tout d'abord, la notion de viabilité ou de développement durable (deux termes employés comme synonymes) est examinée. La définition du rapport Brundtland - " un mode de développement qui répond aux besoins du présent sans compromettre la capacité des générations futures de répondre aux leurs» - est donnée comme exemple parmi «toutes sortes de définitions et de descriptions du développement durable » (ibid., p. 15), tant il est vrai que « les experts eux-mêmes n'ont pas fini d'en débattre » (ibid., p. 8). C'est donc sur un terrain mouvant que l'EAV se construit. La déclaration issue de la Conférence de Thessalonique entérine officiellement la viabilité comme objectif éducatif. Elle «embrasse les questions non seulement d'environnement, mais aussi de pauvreté, de population, de santé, de sécurité alimentaire, de démocratie, de droits de l'être humain et de paix » (ibid., p. 10).

4 En 2002, le Sommet Mondial pour le Développement Durable (SMDD) de Johannesburg est à l'origine de la résolution onusienne proclamant la Décennie de l'éducation pour le développement durable (ci-dessous, DESD) pour la période 2005-2014. L'ESD proprement dite succède ainsi à l'EAV, qui a fait l'objet d'un bilan «d'une décennie de travaux » dans un rapport de l'UNESCO :

Il y a un vrai problème dans la manière dont ces programmes présentent l'environnement et le développement durable - qu'ils relèvent du secteur formel ou informel : on n'y souligne pas assez le rapport entre la santé humaine et la viabilité des écosystèmes ; étudiants et membres de la communauté sont rarement invités à réfléchir aux conséquences de leurs activités, de celles de leurs familles et de l'ensemble de la société sur le fonctionnement des écosystèmes. L'éducation formelle scinde généralement l'enseignement relatif à la société, à l'économie et à l'environnement en disciplines distinctes, au mépris d'apprentissage des formes de compétences pratiques liées à la viabilité. C'est pourquoi l'Action 21 a demandé que l'éducation soit réorientée dans une nouvelle direction. (UNESCO, 2002)

Dans ce rapport est recommandée une "façon nouvelle d'envisager l'éducation », une "éducation innovante", c'est-à-dire «un programme d'étude réorienté vers le développement durable» (ibid., p. 20-21). Le plan international de mise en œuvre de la DESD qui définit l'ESD comme une éducation au service de la durabilité » (UNESCO, 2005a, p.32), fixe alors quatre axes prioritaires: promouvoir l'éducation de base, réorienter et réviser les programmes d'enseignement, informer et sensibiliser le public, dispenser une formation pratique (UNESCO, 2005b).

Insufflée par des injonctions internationales, l'ESD, dont la genèse est très clairement politique, a été "traduite » dans les réformes éducatives de très nombreux pays, tout en intégrant une disparité de points de vue nationaux (Sauvé, Berryman et Brunelle, 2003). Sans attendre la fin de cette décennie, en 2014, nous souhaitons donc, dans les lignes qui suivent, présenter divers contextes de mise en place de l'ESD qui soulignent des approches différentes, notamment en termes de valeurs et de finalités. Cependant, il faut bien reconnaître que cette tentative de présentation tout à la fois synthétique et analytique est très difficile à réaliser : d'une part, parce que les politiques évoluent à ce sujet et d'autre part, car les documents écrits dans de nombreuses langues différentes posent indéniablement des problèmes de traduction. Pour combler les limites de la première partie de cet article, qui ne présente donc pas véritablement de photographie, 
à une époque précise, de la variabilité des approches de l'ESD, nous proposons dans un deuxième temps une succincte analyse diachronique de la politique de l'ESD en France.

7 En reprenant les résultats de Zwang et Girault (2012), nous montrons que l'éducation au développement durable française (nommée ci-dessous EDD) s'inscrit dans des choix éthiques et stratégiques qui prennent corps dans des textes et discours de référence, dont la teneur oscille entre une éducation du futur citoyen et une éducation du futur acteur économique.

\section{Des contextes divers de mise en place de l'ESD}

\section{Des politiques nationales plus ou moins prescriptives}

8 La façon dont les instances nationales ont répondu à la prescription de l'éducation pour le développement durable n'est pas, comme nous souhaitons le démontrer dans cet article, homogène ${ }^{2}$. Si certains pays ont une approche très incitatrice, voire coercitive, d'autres sont beaucoup plus en retrait. L'analyse des diverses réponses du secteur de l'éducation à la recommandation de l'ONU de promouvoir le développement durable amène en effet à observer un gradient de positionnement entre deux pôles (Sauvé, 2006).

9 À l'une des extrémités se trouvent des choix nationaux imprégnés d'une vision du monde éconocentrée, néolibérale, et adoptant une stratégie autoritaire de généralisation. C'est ainsi qu'au Royaume-Uni, en réponse à la Stratégie nationale de développement durable, le "Department for Education and Skills» s'est doté d'un « Sustainable Development Action Plan for Education and Skills » rejoignant les buts fixés par le Department, dont celui de contribuer à "construire une économie nationale compétitive ». L'Angleterre s'est donc dotée du programme « Learning to last » dont le langage et les objectifs renforcent l'une des principales tendances du curriculum global : les apprentissages scolaires doivent préparer les jeunes au marché du travail, en vue du développement économique (Blewitt, 2005 ; Porfilio et Yu, 2006). Comme nous l'avons déjà souligné :

Ici, on retrouve manifestement le paradigme rationnel/technologique de l'éducation. On observe une conception de l'environnement comme ressource à gérer (le capital environnemental) et une vision du développement comme l'atteinte de la prospérité grâce à une économie stable et compétitive. Une telle économie est perçue comme la condition première pour l'amélioration de l'environnement et de la qualité de vie. (Girault et Sauvé, 2008)

Si, à ses prémisses, le modèle français de l'ESD paraissait moins libéral (Sauvé, 2006), nous verrons que les évolutions récentes de son application en France tendent pourtant à s'en rapprocher. À l'autre extrémité, l'état néerlandais ${ }^{3}$ a développé une politique d'ESD moins autoritaire et bien plus diversifiée, qui se traduit notamment par la mise en place de deux programmes pluriannuels. Le « Leren voor Duurzame Ontwikkeling ${ }^{4}$ » (LVDO), qui signifie "apprendre pour le développement durable», destiné aux différents niveaux de l'enseignement ainsi qu'à différentes branches du gouvernement, et le Natuur en Milieueducatie (NME) ${ }^{5}$ qui s'apparente bien plus à une Éducation à la Nature et l'Environnement. Plusieurs réseaux développent en parallèle une ESD plus pratique ou «appliquée». Il s'agit par exemple du "Dutch National Network for Sustainable Development in Higher Education Curricula (DHO)» qui propose des enseignements spécifiques basés sur l'acquisition de savoirs et savoirs-faire ayant in 
fine pour objet d'aider les étudiants à développer des compétences pour tendre vers un développement durable (Wals et Blewitt, 2010), ce qui correspond à une troisième vague d'émergence de l'ESD dans le supérieur, focalisée sur l'enseignement et l'apprentissage de pratiques durables. Du côté de l'enseignement professionnel et de la formation des enseignants existent également des programmes spécifiques, le « Duurzaame MBO » et le « Duurzame PABO » (« Duurzaame» signifiant durable). Cette très riche palette de programmes n'est pas imposée aux enseignants, dans le strict respect de la constitution de ce pays qui leur octroie une liberté académique.

11 Entre ces deux extrêmes, les modalités de mise en œuvre de l'ESD sont plus complexes, à mi-chemin entre les échelons étatique et régional. Nous tentons de rendre compte de cette complexité, à travers différents exemples.

\section{Un gradient de liberté lié à une manière d'administrer et de concevoir l'éducation}

\section{En Pologne}

12 Comme dans tous les autres pays européens, la Stratégie régionale pour l'Europe, adoptée à Vilnius, est officiellement le référentiel de l'ESD polonaise (UNECE, 2005). L'objectif principal du groupe dirigé par Anna Kalinowska est donc la promotion et la diffusion des thèses de la Décennie. Mais Tadeusz Borys (2010) souligne des faiblesses dans la coordination d'actions. Il précise que l'éducation de ce «nouveau paradigme du développement» (p. 61, traduction libre) n'est pas encore mise en place de façon systématique, car les deux ministères qui en sont chargés ${ }^{6}$ ne tiennent pas compte du développement durable. Selon cet auteur, c'est donc le manque d'intérêt et d'encouragement de la sphère politique aux niveaux national, régional et local qui pose problème pour la déclinaison des objectifs de la Décennie, ce qui se traduit, contrairement à d'autres pays, par une absence de plan d'action adopté par le parlement.

\section{En Allemagne}

Dans ce pays, l'éducation est administrée au niveau des Länder. Par conséquent, il n'est pas possible de mettre en place un programme éducatif national. Cependant, les objectifs allemands pour la Décennie sont résumés dans un plan d'action national (UNESCO, 2008), attestant la nécessité et la volonté de la mise en place de l'ESD ${ }^{7}$. Résultant d'un large processus de consultation de la société civile, il formule des objectifs stratégiques et énumère une soixantaine de mesures concrètes pour la promouvoir, tel le programme Transfer 21 (2004-2008), subséquent du programme BLK 21 de la commission Bund-Länder. À côté de ce programme, il existe une multitude d'autres projets s'inscrivant dans l'ESD dans l'enseignement scolaire, notamment les écoles UNESCO, les « Umweltschulen in Europa » (Écoles vertes en Europe, dont le nombre est d'environ 190) et les "GLOBE-Schulen " (Global Learning and Observations to Benefit the Environment), dont le nombre en 2009 était, selon le Ministère allemand de l'éducation et de la recherche, d'environ 470. Jusqu'à l'automne 2008, environ 120 projets scolaires étaient désignés comme projets de la DESD (ibid.), avec une majorité d'écoles primaires. En 2006, l'étude de Rode menée à partir de sites internet de 150 écoles ne participant à aucun projet modèle en matière de développement durable a montré que $10 \%$ des 
écoles affichaient cependant des indications claires sur les aspects de la durabilité (en référence aux trois piliers: économie/écologie/social). C'est toutefois la dimension écologique qui semble jouer un rôle central, les dimensions sociale et économique restant en marge (ibid.).

Compte tenu de l'originalité du principe directeur du Transfer 21 et du concept (non traduisible) de «Gestaltungskompetenz $z^{8}$ » qui lui est spécifique, présentons plus en détail l'ensemble de ce programme. Celui-ci avait pour objectif de diffuser et d'ancrer systématiquement les résultats du précédent programme BLK 21 dans le cadre d'un transfert vers les écoles de l'enseignement général, tout en s'adaptant aux structures et aux réseaux préexistants dans les Länder respectifs. Afin d'ancrer l'ESD à grande échelle, en plus des établissements de l'enseignement secondaire, les écoles primaires, les "Ganztagsschulen " " et les centres de formation initiale et continue des enseignants ont été intégrés au Transfer 21 (Programm Transfer 21, 2004-2008, Rapport final). Dans ce cadre, l'ESD a, entre autres, servi à l'élaboration de normes de qualité et à la coopération entre des partenaires extérieurs et le monde scolaire. Ainsi, jusqu'en juillet 2008, plus de $10 \%$ des établissements scolaires des 14 Länder participants ont été impliqués dans le programme Transfer 21, soit 2586 établissements. Il convient de noter qu'on distingue trois niveaux d'implication au programme : le travail intensif sur l'ESD (238 établissements), la réalisation de projets (1421 établissements), et l'implication de certains enseignants (927 établissements) (ibid.). La majorité des établissements a mis en place des projets ou des activités relatives aux questions du développement durable. Une véritable évaluation comparative du niveau d'implication est tout de même difficile, car les critères évaluatifs diffèrent selon les Länder.

Selon Gerhard de Haan, Président du Comité national de la Décennie des Nations Unies, l'objectif principal de l'ESD en Allemagne est la transmission de la Gestaltungskompetenz. Ce concept a été développé et testé à la Freie Universität Berlin dans le cadre du programme BLK-21 :

L'éducation au développement durable est une nouvelle vision durable de l'éducation qui met au centre le développement des compétences et des savoir-faire nécessaires pour l'action participative. Les connaissances enseignées aident les apprenants à déterminer activement et de façon responsable des options/ possibilités d'actions durables. Ces compétences peuvent être regroupées sous le terme de la Gestaltungskompetenz » (de Haan et Harenberg, 1999 ; cité par le BMBF, 2009, traduction libre).

La Gestaltungskompetenz se réfère aux domaines des compétences clés de l'OCDE. On distingue douze compétences spécifiques de la Gestaltungskompeten $z^{10}$ :

1. Développer son savoir en étant ouvert sur le monde et en intégrant de nouvelles perspectives;

2. Savoir réfléchir et agir de façon anticipatoire ;

3. Acquérir des connaissances et agir dans une démarche interdisciplinaire ;

4. Savoir planifier et agir en collaboration avec d'autres acteurs;

5. Savoir participer à des processus décisionnels ;

6. Être capable de motiver d'autres personnes à devenir actives ;

7. Être capable de réfléchir à ses propres conceptions du monde et à celles des autres;

8. Savoir planifier et exécuter son action de manière autonome ;

9. Savoir faire preuve d'empathie et de solidarité envers des personnes défavorisées ;

10. Savoir se motiver à devenir actif ; 
11. Savoir utiliser ses perceptions de la justice comme base de prise de décision et d'action;

12. Montrer de l'empathie pour autrui.

17 Le concept de Gestaltungskompetenz est donc principalement axé sur l'acquisition de compétences pour construire et comprendre une modélisation active de l'avenir. Celuici diffère donc très notablement d'une simple approche comportementaliste. Selon de Haan, il s'agit d'« une incitation à travailler activement à des objectifs d'avenir dans le sens du développement durable : "il s'agit d'avoir soi-même des idées (...) et de pouvoir aussi [les] mettre en œuvre" " (extrait d'entretien cité dans Nagel U. et coll., 2008). Dans ce contexte, l'avenir est ouvert et compris comme " gestaltbar », soit littéralement un " possible d'être créé » (ibid.). Cet avenir peut être pensé par des individus, c'est-àdire modélisé à travers un engagement dans l'action. Selon certains protagonistes, la Gestaltungskompetenz comprend une composante utopiste et visionnaire ainsi qu'une réflexion anticipatoire, travaillée sur la base de prévisions, de scénarios, de simulations, d'évaluation des technologies et de calculs de risques. Dans le document de présentation du programme Transfer 21 , de Haan précise qu'un changement culturel est nécessaire afin d'agir dans le sens du développement durable (Programm Transfer 21, Document de présentation, n.d.).

En Allemagne, l'ESD a donc pour finalité première d'acquérir la Gestaltungskompetenz, pour in fine faire des choix rationnels et argumentés, anticiper, gérer différentes perspectives des parties prenantes et identifier l'équilibre entre différentes valeurs. Il s'agit d'appliquer le savoir sur le développement durable et de reconnaître les problèmes résultants $d u$ développement non durable. C'est-à-dire tirer des analyses actuelles et des études prospectives des conclusions sur les évolutions environnementales, économiques et sociales dans leur dépendance mutuelle, et sur cette base comprendre et mettre en œuvre les décisions pour réaliser des processus du développement durable. (Ibid., p. 19, traduction libre)

Pour atteindre cet objectif, trois principes sont privilégiés : l'enseignement des savoirs interdisciplinaires, des formes participatives d'apprentissage et l'établissement de structures innovatrices. La coopération avec des partenaires extrascolaires est dans ce contexte très importante (ibid.).

Malgré les perspectives qu'offre la Gestaltungskompetenz, nous souhaitons tout de même en souligner deux limites. Premièrement, il nous semble qu'elle ne laisse pas d'espace à d'autres visions du monde que celle centrée sur une pensée positiviste: nos connaissances scientifiques nous permettront de régler tous les problèmes inhérents à un développement important de nos sociétés. Deuxièmement, elle ne permet pas un recul critique face à l'injonction du développement durable. En effet, comme nous l'avons déjà analysé au sein des Travaux d'Initiative Personnelle Encadrés en France (Girault, 2012), les apprenants ont la possibilité de choisir parmi différentes options, mais toujours et seulement « dans le sens du développement durable ». Ne serait-il pas alors pertinent d'inclure à ce programme un champ d'investigation portant sur les enjeux philosophiques liés aux progrès techniques?

\section{En Suisse}

20 Le gouvernement suisse a développé, depuis 1997, une «Stratégie pour le développement durable ", actualisée en 2002, puis en 2008. Elle fixe les priorités de la politique menée par le Conseil fédéral en matière de développement durable. Il est en premier lieu intéressant de préciser que le développement durable est à ce jour inscrit 
dans la Constitution fédérale. L'article 2 le place au rang de but constitutionnel et l'article 73 (Développement durable) demande à la Confédération et aux cantons d'œuvrer «à l'établissement d'un équilibre durable entre la nature, en particulier sa capacité de renouvellement, et son utilisation par l'être humain ». Cependant, à l'instar de l'Allemagne qui a un système d'enseignement décentralisé, il n'existait pas initialement en Suisse d'organe de coordination chargé de la mise en œuvre de l'ESD au niveau national. Comme l'éducation relève de la juridiction cantonale, environ 26 systèmes éducatifs y cohabitent, ce qui constituait un obstacle pour la généralisation de l'ESD $^{11}$, qui jusqu'alors avait fait l'objet de mesures de soutiens ponctuels, ne s'inscrivant toutefois pas dans une cohérence d'ensemble. L'ESD restait en effet une option choisie par certaines personnes, certains groupes ou institutions convaincus et engagés. Dans le système formel, la Décennie ne semblait concerner que quelques disciplines des sciences de la nature (environnement) et des sciences humaines. Enfin, la formation initiale étant trop centrée sur les disciplines, seuls certains formateurs, comme ceux de géographie, se sentaient investis de la tâche de former les enseignants en intégrant le développement durable, mais dans une vision qui n'unissait pas forcément avec tous les aspects transversaux de l'ESD. Depuis 2008, la Conférence suisse de coordination de l'éducation au développement durable réunissant des directeurs cantonaux de l'instruction publique (CDIP) est chargée de coordonner l'intégration de l'ESD dans l'enseignement ${ }^{12}$. Celle-ci assure le pilotage politique avec les 4 conférences régionales et les offices fédéraux. Le «Plan de mesures 2007-2014, Éducation au développement durable » vise notamment à soutenir l'intégration du développement durable dans les plans d'études, la formation des enseignants. La Conférence suisse de coordination de l'éducation au développement durable a aussi conduit à la création d'une agence spécialisée en janvier 2013.

\section{En Italie}

Dans ce pays ${ }^{13}$, ce sont les instances centrales qui rédigent les textes de référence en matière de développement durable et d'ESD : le Ministère de l'environnement et de la tutelle du territoire et de la mer et le Ministère de l'éducation, de l'université et de la recherche. En 2009, dans la charte "École, environnement et légalité $»^{14}$, ces deux ministères ont entériné l'entrée de l'éducation environnementale et au développement durable dans un enseignement déjà préexistant (Citoyenneté et Constitution). Cette éducation est intégrée comme un "thème interdisciplinaire et transversal, une aire d'apprentissage déterminée par l'intersection de nombreuses matières se caractérisant par leur spécificité de contenus et connexions interdisciplinaires.» (Extrait de la charte ${ }^{15}$, traduction libre)

Les projets mis en place par ces deux institutions ont comme objectif de développer chez les jeunes générations :

- la compréhension des problématiques naturelles, du paysage, culturelles de l'environnement et du territoire dans lesquelles les jeunes générations vivent ;

- la prise de conscience qu'il est possible de respecter, conserver, protéger et améliorer l'environnement et le territoire en élaborant des projets d'intervention et en les proposant à l'école et à d'autres institutions des communautés d'appartenance ;

- la réflexion sur la valeur de l'air, de l'eau, de la terre en tant que bien commun et comme droit universel pour relancer dans l'école, dans la famille et dans tous les milieux des 
comportements de consommation durable de ces biens, en ayant soin de les protéger et de les développer, au service aussi des générations à venir. soutenabilité en mesure de former les citoyens à des choix conscients et éthiques en ce qui concerne la consommation, les modes de vie, le transport, l'épargne énergétique, la réduction et le tri des déchets, et de façon plus générale, le respect de l'environnement» (Extrait du guide pour l'éducation à l'environnement et au développement durable, signé des deux ministères cités plus haut ${ }^{16}$, traduction libre). Cependant, en Italie, il y a 20 régions, dont 5 à statut spécial (Val d'Aoste, Trentin-HautAdige, Frioul-Vénétie Julienne, Sardaigne et Sicile), et chacune de ces régions applique de façon autonome les législations européennes et nationales de l'ESD. Les projets mis en place y sont donc hétérogènes et pour l'instant aucune étude n'a été, à notre connaissance, mise en œuvre afin d'analyser la façon dont sont intégrées les prérogatives nationales.

\section{Les pays latino-américains}

Enfin, pour compléter cette présentation partielle de l'éventail des contextes de mise en place de l'ESD, se trouvent plusieurs pays latino-américains (dont le Brésil, la Colombie, Cuba, le Mexique et le Venezuela), qui proposent des politiques nationales plus critiques et distantes de la prescription onusienne. Ainsi, le lancement sousrégional de la DESD pour l'Amérique latine a eu lieu à l'occasion de la Conférence ibéroaméricaine sur le développement durable tenue à Rio de Janeiro, Brésil, en juin 2005, et le lancement sous-régional pour les Caraïbes pendant la conférence sur «L'éducation au service du développement durable: de nouvelles approches pour l'avenir" organisée à Kingston, Jamaïque, en octobre 2005 (UNESCO, 2005c). Lors de la réunion de mobilisation régionale sur le thème «Bâtir l'éducation au service du développement durable en Amérique latine et dans les Caraïbes ", qui a eu lieu à San José, Costa Rica, en octobre 2006, l'UNESCO et la Charte de la Terre ont conjointement souligné la nécessité d'une stratégie régionale pour la DESD. C'est ainsi qu'en se basant sur une riche tradition d'ERE, où le rapport à l'environnement a toujours été envisagé dans une perspective socioécologique (alimentation, pauvreté, diversité culturelle, droits humains), González-Gaudiano (2005) souligne que l'approche des réalités environnementales y est critique, politique et transformative. De fait, le document de stratégie reconnaît que le concept de durabilité est à même de créer des synergies entre différents thèmes éducatifs, relatifs notamment à l'environnement, l'interculturalité, la paix, les droits humains, la réduction de la pauvreté, la santé, le VIH/SIDA, l'alphabétisation et l'égalité des sexes face à l'éducation. Dans cette région, l'ESD se fonde donc sur la vision commune que les politiques éducatives devraient contribuer à enrayer les processus aigus de dégradation et destruction de l'environnement, ainsi qu'à édifier des sociétés plus justes.

Après avoir présenté diverses modalités de mise en place de la politique onusienne de l'ESD, nous souhaitons montrer que cela est bien plus complexe puisqu'au sein de chaque pays les politiques de mise en place de l'ESD peuvent évoluer. Pour illustrer ce propos, présentons l'évolution de la généralisation de l'éducation au développement durable en France. 


\section{La mise en place et l'évolution de l'Éducation au développement durable (EDD) en France (2003-2011)}

L'étude d'une cinquantaine de documents relatifs à l'EDD ${ }^{17}$ en France ordonnés, pour l'analyse, selon leur valeur normative en droit français, c'est-à-dire entre textes officiels à valeur de $\operatorname{loi}^{18}$ (désigné «lettre» de l'EDD) et textes non contraignants juridiquement ${ }^{19}$ (dénommé « l'esprit »), a permis à Zwang et Girault (2012) de dégager une évolution selon deux grandes périodes, distinguées essentiellement d'un point de vue téléologique.

\section{De 2003 à 2008 : I'EDD prescrite dans une perspective citoyenne}

Après une phase d'expérimentation au cours de l'année 2003-2004, la rentrée 2004 a été marquée par une injonction ministérielle relative à la "généralisation d'une éducation à l'environnement pour un développement durable [EEDD] » (MEN, 2004). Trois ans plus tard, un nouveau plan en faveur de l'éducation au développement durable a été lancé (MEN, 2007). Les objectifs stratégiques des deux circulaires sont en continuité : inscrire plus largement cette éducation dans les programmes d'enseignement, multiplier les démarches globales d'EDD dans les établissements et les écoles, former les professeurs et les autres personnels impliqués dans cette éducation.

Les deux premières circulaires s'inscrivent dans la première « Stratégie nationale pour le développement durable » (SNDD, 2003-2008), cadre de la politique nationale de l'EDD, dont la première version de 2003 donne une large part à la formation citoyenne. Les finalités éducatives affichées et qui doivent être appliquées, sont celles de l'école républicaine, caractéristique d'une "citoyenneté à la française " (Tutiaux-Guillon, 2006), soit former des citoyens autonomes, responsables, libres de leur choix, ce que montre cet extrait de programme de SVT (Sciences de la Vie et de la Terre) :

L'objectif, pour le professeur, est d'éduquer au choix et non d'enseigner des choix réputés meilleurs que d'autres. L'éducation à la santé et celle au développement durable sont l'occasion d'amener l'élève à prendre conscience que les sujets abordés soulèvent des questions d'éthique et à acquérir responsabilité et autonomie. (MEN, 2008, p. 34)

Cependant, dans les textes non officiels et fondateurs, ce libre choix est circonscrit dès le départ dans une éthique environnementale restreinte, soit l'anthropocentrisme qui est tout d'abord mis en avant dans une perspective humaniste :

Le souci de développement durable conduit à replacer l'être humain au centre de toutes les préoccupations. Ce qui confère au pilier social une dimension spécifique. Ainsi ne peut-il y avoir de développement durable sans dignité de l'homme et de la femme, ni sans égalité entre eux. (Comité interministériel pour le développement durable, 2003, p. 5)

L'anthropocentrisme est aussi considéré comme un moyen de favoriser l'interdisciplinarité par l'approche gestionnaire qu'il induit :

Le concept de développement durable aide d'ailleurs peut-être à accrocher l'analyse de l'environnement sur une idée plus immédiate de " gestion de l'envi-ronnement » dans lequel l'homme n'occupe pas obligatoirement la position de coupable et de destructeur. » (Bonhoure et Hagnerelle ${ }^{20}, 2003$, p. 13)

31 Progressivement, cette approche gestionnaire va s'imposer dans les programmes. 


\section{À partir de 2008 : I'EDD dans une perspective économique} dans la continuité des négociations du Grenelle de l'environnement, évoque pour la première fois la nécessaire émergence de "compétences Développement durable" (sic) (Bregeon, Faucheux, et Rochet, 2008, p. 8). Tout en indiquant qu'il faudra les définir, en les associant plus volontiers au milieu professionnel, il indique cependant que l'école forme de futurs professionnels : « les entreprises sont en demande de jeunes formés au développement durable ou plus précisément de jeunes issus de formations les conduisant à des métiers concourant au développement durable.» (Ibid., p. 10). Cette quête d'efficience économique de la formation scolaire est reprise dans la nouvelle SNDD «Vers une économie verte et équitable ( Comité interministériel pour le développement durable, 2010) qui fixe parmi ses objectifs stratégiques prioritaires en éducation :

- la lutte contre le décrochage scolaire, qui «doit permettre à l'ensemble des jeunes de s'insérer rapidement dans la société et de faire face à ses mutations et aux évolutions du contexte économique » (p. 13) ;

- le développement de compétences et des qualifications dans six secteurs prioritaires: bâtiment, énergie, agriculture, sciences pour la mer, l'économie et la santé;

- le développement des métiers et les filières de l'environnement, du recyclage, de l'écoconception, des analyses du cycle de vie et de la con-naissance des écosystèmes.

Ces changements d'objectifs stratégiques dans les textes non officiels aboutissent en 2011 à la publication de la troisième circulaire EDD, dont le cadre de référence est la SNDD de 2010, le Grenelle de l'environnement, le Grenelle de la mer et le plan national de mobilisation des métiers et des formations de la croissance verte. Alors que, dans ce texte officiel, ne sont écrits que les objectifs stratégiques des deux circulaires précédentes et que la finalité éducative annoncée est toujours l'éducation au choix face à des problèmes complexes, le cadre de référence informe sur la finalité économiciste sous-jacente.

Or ceci illustre bien ce que Bertrand et Valois (1999) affirment lorsqu'ils écrivent que les fins réelles d'une éducation sont inscrites dans les champs paradigmatiques et politiques de la société, alors même que les fins déclarées sont autres : «il ne suffit pas [...] de se fier aux perceptions et aux déclarations des interlocuteurs de l'organisation éducative ; il importe de repérer la quelconque volonté extérieure, à savoir les forces qui dictent les fins réelles de l'organisation éducative» (p. 25). Les textes non officiels sont à cet effet très informatifs. Les objectifs français se sont ainsi progressivement rapprochés d'un cadre libéral, comme nous l'avons déjà décrit pour le Royaume-Uni, et ceci en cohérence avec la Stratégie Européenne de Développement Durable.

\section{Conséquences du point de vue des rapports aux savoirs}

L'éthique clairement anthropocentrique et les changements dans les objectifs ont été traduits dans les savoirs à transmettre inscrits dans les programmes et les référentiels de formation professionnelle. Les programmes de Sciences de la Vie et de la Terre (SVT) et de géographie n'ont pas été affectés au même moment par l'EDD. Une première vague de changement a concerné les SVT au collège entre 2005 et 2008, tandis qu'une 
deuxième vague a touché les programmes de géographie en 2009 au collège, et au lycée en SVT et en géographie à partir de 2010. Les thèmes affichés comme étant le plus en lien avec l'EDD sont l'énergie (en $5^{\mathrm{e}}, 2^{\mathrm{e}}$ en géographie et en $3^{\mathrm{e}}$ et $2^{\mathrm{e}}$ en SVT), la biodiversité (en $6^{\mathrm{e}}, 5^{\mathrm{e}}, 4^{\mathrm{e}}$ et $2^{\mathrm{e}}$ en SVT), le sol (en $6^{\mathrm{e}}$ et en $2^{\mathrm{e}}$ en SVT), les ressources (eau, air, halieutiques, alimentation) en $5^{\mathrm{e}}$, et le développement durable en géographie en $2^{\mathrm{e}}$ et en $5^{\mathrm{e}}$. Ce dernier devient dès lors un objet d'enseignement parmi d'autres à partir d'une conception admise par l'institution, tangible dans les documents d'accompagnement des programmes de géographie : «C'est le bien-être des hommes en société qui est l'enjeu du développement durable et non la préservation d'une nature « déifiée ou sanctuarisée » (DGESCO - IGEN, 2010, p. 1).

On constate en étudiant les thèmes de ces nouveaux programmes que le référentiel anthropocentrique, qui était déjà observé au sein des anciens programmes s'est progressivement affirmé, en prenant une orientation de plus en plus utilitariste, éthique qui ne donne de la valeur à l'environnement que par rapport au bien-être qu'il procure (Birnbacher, 1998, p. 429). En effet, les contenus des deux disciplines, tels qu'ils sont rédigés, s'orientent de plus en plus vers les besoins humains, la gestion des ressources et les actions à mener. Les changements les plus importants ont lieu en géographie, avec des indications précises sur le cadre théorique à adopter pour l'étude des interactions entre humains et nature: "Si on l'envisage comme écosystème, le littoral correspond à l'estran, c'est-à-dire à la zone de contact, éminemment mobile, entre trois éléments : l'air, la terre et la mer. Mais la question élargit la perspective et définit le littoral comme un "anthroposystème"." (DGESCO - IGEN, 2010, p.1). Corrélativement, des notions scientifiques descriptives et explicatives ont tendance à s'effacer. Par exemple, les explications des causes du changement climatique qui étaient clairement présentes dans l'ancien programme de 2nde sont désormais réservées aux seuls élèves qui atteindront la spécialité SVT en Terminale (année du baccalauréat). Cette tendance à l'orientation des savoirs et à la réduction du champ épistémique est observée dans d'autres contextes, universitaires et non formels (Barthes, Zwang, et Alpe, dans ce numéro). Parallèlement, de nouvelles formations et de nouveaux référentiels en baccalauréat professionnels et technologiques émergent. La plus emblématique est la filière STI2D (Sciences et Technologie de l'Industrie et du Développement Durable) créée en 2011 dont la visée est la formation des jeunes en vue d'une économie verte :

[La série] vise l'acquisition d'une formation technologique polyvalente renforcée par un enseignement technologique spécifique décliné en quatre domaines: énergie et environnement, systèmes d'information et numérique, innovation technologique et éco-conception, architecture et construction. Ainsi redéfinie, la série STI2D permet de mieux prendre en compte les préoccupations liées au développement durable, et notamment les préconisations formulées lors de la Conférence nationale sur les métiers de la croissance verte qui s'est tenue dans le cadre du Grenelle de l'environnement. (MEN - DGESCO, 2011)

37 Nous pensons que l'orientation de ces formations et des savoirs qui y sont associés sont nés d'un collapsus entre objectifs éducatifs et objectifs stratégiques, les uns étant au service des autres. Il est d'ailleurs intéressant de noter que conjointement, dans la troisième circulaire EDD, alors que pléthore d'objectifs stratégiques sont décrits, le champ pédagogique est très peu investi, que ce soit dans les approches pédagogiques ou dans les objectifs éducatifs, hormis le développement de compétences du socle commun. 

seule réponse aux problèmes sociaux et environnementaux, il nous semble que l'éducation nationale française participe à la naissance d'une forme de politique écologique focalisée sur les besoins humains et la gestion des ressources (Zwang et Girault, 2012). Tout cela étant présenté comme "naturel » au sein du développement durable. Or, comme l'affirment Michel Soëtard et Renaud Hétier, "la finalité [en éducation] ne doit pas cesser d'être pensée sous l'égide de la liberté »; autrement dit, en éducation, il s'agit moins de «modeler selon une idée préconçue, que de faire produire à la matière humaine même sa forme en liberté, sa forme qui est liberté. » (2003, p. 64). Le choix d'une éthique individuelle et d'un projet politique sont des libertés et la réduction du champ des possibles n'est pas signe d'une " éducation au choix », pourtant leitmotiv institutionnel exprimé au sein des textes prescripteurs de l'EDD.

\section{En guise de conclusion}

Nous avons souhaité, dans le cadre de cet article, montrer que contrairement à une représentation commune, la mise en place de l'ESD au niveau international ne s'effectue pas de façon homogène. S'il est totalement souhaitable d'observer, dans le cadre de la mise en place de programmes d'enseignement, des disparités locales liées à des contextes socio-éducationnels spécifiques, il nous semble que la très grande variabilité des approches décrites traduit bien plus des divergences fortes d'analyse et d'appropriation de l'ESD. Ainsi, contrairement à ce que certains prescripteurs de programmes pourraient ou souhaiteraient laisser entendre, la prescription internationale de l'ESD et ses applications locales sont de fait l'objet de choix idéologiques et politiques très importants qui divergent donc naturellement selon les pays et les époques. Il est d'ailleurs très intéressant de noter la très grande neutralité, sur cet aspect, des Hollandais qui inscrivent dans leur propre constitution la liberté des choix pédagogiques des enseignants, ce qui a pour conséquence le fait d'offrir des outils aux enseignants pouvant les aider à initier des enseignements très divers privilégiant pour certains des approches naturalistes (bio et écocentrée) et pour d'autres des approches sociocentrées ou plus inscrites dans l'ESD soit clairement anthropocentrée. Il est également intéressant de noter que parallèlement à la vision très utilitariste de l'environnement prônée par l'ESD, certains pays qui privilégient cette approche tendent vers une vision également très utilitariste de l'école qui ne doit plus exclusivement tendre à enseigner des savoirs fondamentaux, mais plus fondamentalement à préparer les jeunes au marché du travail.

\section{BIBLIOGRAPHIE}

Bertrand, Y., Valois, P. (1999). Fondements éducatifs pour une nouvelle société. Lyon : Chronique Sociale. Montréal : Éditions nouvelles.

Éducation relative à l'environnement, Volume 11 | 2014 
Blewitt, J. (2005). Education for Sustainable Development, Governmentality and Learning to Last. Environmental Education Research, 11(2), 173-185.

Birnbacher, D. (1998). Éthique utilitariste et éthique environnementale - une mésalliance ? Revue Philosophique de Louvain, 96(3), 427-448.

Borys, T. (2010). Decade of Education for Sustainable Development - Polish Challenges. Problems of sustainable development, 5(1), 59-70.

Girault, Y. et Sauvé, L. (2008). L'éducation scientifique, l'éducation à l'environnement et l'éducation pour le développement durable : Croisements, enjeux et mouvances. Dans Girault, Y. et Sauvé, L. (dir.). L'éducation à l'environnement ou l'éducation au développement durable, 46, 7-30.

Girault, Y. (2012). Environnement et développement : présupposés épistémologiques et retombées éducatives des Travaux d'Initiative Personnelle Encadrés. Dans Bader, B. et Sauvé, L. (dir.). Éducation, Environnement et Développement Durable : vers une écocitoyenneté critique (p. 313-342). Laval : Presses de l'Université Laval.

Gonzalez-Gaudiano, E. (2005). Education for Sustainable Development : Configuration and meaning Policy Futures in Education. Environmental Education And Education For Sustainable Development, 3(3), 243-250.

Harenberg, D. (2002). Bildung für eine nachhaltige Entwicklung in der Wissensgesellschaft. Thèse de doctorat inédite. Freie Universität Berlin.

Nagel, U., Kern, W. et Schwarz, V. (2008). Contributions à la définition de compétences et de standards pour l'éducation en vue du développement durable sous l'angle de l'éducation à l'environnement, de l'éducation à la santé et de l'éducation dans une perspective globale. Forschung und Entwicklung, Pädagogische Hochschule Zürich. Récupéré du site : www2.phzh.ch/ ForschungsDB/Files/167/Etude_competences_edd.pdf

Porfilio, B.J. et Yu, T. (2006). Student as Consumer : a critical narrative of the commercialization of teacher education. Journal for Critical Education Policy Studies, 4(1).

Sauvé, L., Berryman, T. et Brunelle, R. (2003). Environnement et développement : la culture de la filière ONU. Éducation relative à l'environnement : Regards - Recherche - Réflexions, 4, 33-55.

Sauvé, L. (2006). L'organisation et la structuration du secteur de l'éducation en rapport avec les énoncés du développement durable. Liaison Énergie - Francophonie - « Former et éduquer pour changer nos modes de vie ", 72, 33-41.

Soëtard, M. et Hétier, R. (2003). L'éducation entre fin, finalité et finalisme. Le sens de la pédagogie : repères philosophiques, résonances pédagogiques. Revue Française de Pédagogie, 143, 61-68.

Tutiaux-Guillon, N. (2006). Le difficile enseignement des « questions vives » en histoiregéographie. Dans Legardez, A. et Simonneaux, L. (dir.). L'école à l'épreuve de l'actualité. Enseigner les questions vives (p. 119-135). Paris : ESF Éditeur.

Wals, A. E. J. et Blewitt, J. (2010). Third wave sustainability in higher education : Some (inter)national trends and developments. Dans Jones, P., Selby, D. et Sterling, S. (dir.). Green Infusions : Embedding Sustainability across the Higher Education Curriculum. London : Earthscan.

Zwang, A. et Girault, Y. (2012). Quelle(s) spécificités pour l'Éducation au Développement durable (EDD) ? Dans Pagoni, M. et Tutiaux-Guillon, N. (dir.). Les éducations à... : nouvelles recherches, nouveaux questionnements, 50,181-195. Éléments du corpus étudié, cités dans l'article 
Bonhoure, G. et Hagnerelle, M. (2003). L'éducation relative à l'environnement et au développement durable. Rapport de l'inspection générale. Récupéré du site de l'éducation nationale : www.education.gouv.fr

Bundesministerium Für Bildung und Forschung (BMBF). (2009). Bericht der Bundesregierung zur Bildung für eine nachhaltige Entwicklung. Récupéré du site du Ministère de l'éducation et de la recherche : www.bmbf.de/pub/bericht_fuer_nachhaltige_entwicklung_2009.pdf

CNUED. (1992). Chapitre 36 - Promotion de l'éducation, de la sensibilisation du public et de la formation. Récupéré du site : www.un.org/french/ga/special/sids/agenda21/action36.htm

Comité interministériel pour le développement durable. (2003). Texte de la SNDD originelle. Récupéré du site du Ministère de l'environnement et du développement durable (MEDD) : www.legrenelle-environnement.fr/IMG/CD_SNDD/IIISNDD_2003-2008/1.SNDD_adoptee_le_3_juin_2003/SNDD-2003.pdf

Comité interministériel pour le développement durable. (2010). Stratégie Nationale de Développement Durable - 2010-2013 - Vers une économie verte et équitable. Récupéré du site du MEDD : www.developpement-durable.gouv.fr/IMG/pdf/SNDD_index.pdf

Deutsche UNESCO-Kommission/Nationalkomitee für die UN-Dekade. (2008). Nationaler Aktionsplan für die UN-Dekade Bildung für nachhaltige Entwicklung. Récupéré du site de la commission allemande de la DESD

DGESCO - IGEN. (2010). Géographie $2{ }^{\mathrm{e}}$ : Sociétés et développement durable, Thème 4 : Gérer les espaces terrestres. Question au choix : les littoraux, espaces convoités. Récupéré du site de l'éducation nationale : media.eduscol.education.fr/file/lycee/75/5/LyceeGT_Ressources_

HGEC_2_Geo_09_T4LittorEspcConv_148755.pdf

DGESCO-IGEN. (2011). Préparation de la rentrée 2011. Récupéré du site : www.education.gouv.fr/ cid55941/mene1111098c.html

Kalinowska, A. (2008). Edukacja dla Zrównowa onego Rozwoju w Polsce-stan i wyzwania w wietle ustale mi dzynarodowych podj tych na IV Konferencji UNESCO nt Edukacji Ekologicznej. Récupéré du site du centre régional de l'éducation écologique de Plock : www.rceeplock.nazwa.pl/files/rcee/ mater_szkol/2_edukacja.pdf

MEN. (2004). Généralisation d'une éducation à l'environnement pour un développement durable. Récupéré le 6 mars 2011 du site : www.education.gouv.fr/bo/2004/28/MENE0400752C.htm

MEN. (2007). Seconde phase de généralisation de l'éducation au développement durable. Récupéré le 6 mars 2011 du site : www.education.gouv.fr/bo/2007/14/MENE0700821C.htm MEN. (2008).

Programme de sciences de la vie et de la Terre - Programmes du collège. Récupéré du site de l'éducation nationale : www.media.education.gouv.fr/file/special_6/52/9/ Programme_SVT_33529.pdf

Programm Transfer 21 (n.d). Document de présentation. Eine Handreichung zur Bildung für nachhaltige Entwicklung Hintergrund - Methoden - Schulpraxis. Récupéré du site du programme Transfer 21 : www.transfer-21.de/daten/materialien/Handreichung.pdf

Programm Transfer 21, 2004-2008. Rapport final. Récupéré du site du programme Transfer 21 : www.transfer-21.de/daten/T21_Abschluss.pdf

UNESCO-PNUE. (1987). Stratégie internationale d'action en matière d'éducation et de formation relatives à l'environnement pour les années 1990. Congrès international sur l'éducation et la formation relatives à l'environnement. Récupéré du site de l'ONU : unesdoc.unesco.org/images/ 0008/000805/080583fo.pdf 
UNESCO. (1997). Déclaration de Thessalonique. Conférence internationale - Environnement et société : Éducation et sensibilisation du public à la viabilité. Récupéré sur le site de l'institution : unesdoc.unesco.org/images/0011/001177/117772fo.pdf

UNESCO. (2002). Éducation pour un avenir viable : Enseignements tirés d'une décennie de travaux, depuis Rio de Janeiro jusqu'à Johannesburg. Récupéré du site de l'institution : unesdoc.unesco.org/images/ 0012/001271/127100f.pdf

UNESCO. (2005a). Décennie des Nations Unies pour l'Éducation en vue du Développement Durable (2005-2014). Plan international de mise en œuvre. Récupéré du site de l'institution : unesdoc.unesco.org/images/0014/001486/148654f.pdf

UNESCO. (2005b). L'UNESCO et le développement durable. Récupéré du site de l'institution : unesdoc.unesco.org/images/0013/001393/139369f.pdf

UNESCO. (2005c). Amérique latine et Caraibes / Éducation / Organisation des Nations Unies pour l'éducation, la science et la culture. Récupéré du site : www.unesco.org/new/fr/ education/themes/ leading-the-international-agenda/education-for-sustainable-development/around-the-world/ latin-america-and-the-caribbean/

UNESCO. (2008). Rapport national soumis au nom du Gouvernement de la Suisse conformément au cadre mis en place par l'UNESCO pour le suivi et l'évaluation de la Décennie des Nations Unies pour l'éducation au service du développement durable. Récupéré du site de la commission suisse pour l'UNESCO : www.unesco-nachhaltigkeit.ch/fileadmin/documents/Questionnaire_DEDD_Suisse_2008.pdf

\section{NOTES}

1. Dans cet article, nous employons l'acronyme ESD (Education for Sustainable Development) pour rendre compte du contexte international, tandis que l'acronyme EDD (Éducation au Développement Durable) est réservé au contexte francophone. Cette distinction nous semble nécessaire pour bien marquer les différentes traductions nationales de l'ESD.

2. Voir à ce sujet les réponses d'une enquête effectuée par l'Unesco dans le cadre de la « Decade on Education for Sustainable Development: Report on implémentation of the UNECE strategy for education for sustainable development »: 26 pays ont répondu parfois de façon succincte à cette enquête qui donne cependant des informations pertinentes : www.unece.org/env/esd/Implement.Gov.htm

3. Nous remercions notre collègue Sabine Von der Hoorn, qui a accepté de rechercher et traduire des documents se référant à la situation en Hollande.

4. Le contenu de ce programme est expliqué en anglais en quelques lignes sur la page : www.senternovem.nl/Leren_voor_duurzame_ontwikkeling/english.asp. Et la brochure en pdf donne les grands axes de ce programme : www.senternovem.nl/mmfiles/LvDo_ programmabrochure_engelstalig_tcm24-290166.pdf

5. www.nme.nl. Voir aussi la fondation « Kennisnet " (qui signifie « réseau de connaissances») qui fait de l'expertise dans tous les niveaux d'éducation. Introduction en anglais sur about.kennisnet.nl/. Il y a plusieurs dossiers consultables sur internet, avec des préconisations pour l'EDD au primaire, secondaire et universités, dont duurzaamheid.kennisnet.nl/.

6. Ministère de l'éducation nationale (MEN) et Ministère de l'enseignement supérieur (MNiSW). 7. La première publication officielle relative à l'éducation pour le développement durable (l'ESD en Allemagne) était le "Cadre d'orientation de l'éducation pour le développement durable ", présenté par la commission Bund-Länder pour la planification de l'éducation et la promotion de la recherche (BLK) en 1998. Elle a été suivie par la publication «L'éducation pour le développement durable - expertise sur le programme », constituant la base pour le programme 
quinquennal BLK-21, crée en 1999. Environ 200 établissements de 15 Länder ont participé au projet.

8. Le terme Gestatungskompetenz s'appuie sur la formulation de la connaissance comme capacité à agir et comme une façon de «bouger quelque chose». (Harenberg, 2002, p. 256).

9. Soit les établissements qui accueillent les élèves durant la journée entière alors que dans les écoles « classiques » l'accueil est limité à la matinée.

10. Les compétences spécifiques sont traduites librement (www.transfer21.de).

11. Voir la Stratégie pour le développement durable : lignes directrices et plan d'action 2008/2011

12. Voir le site de la Fédération suisse d'éducation pour l'environnement (consulté le 7 novembre 2012). Depuis le 1er janvier 2013, c'est la fondation « éducation21» qui a pris le relais et assure partiellement la mise à jour du portail suisse d'éducation à l'environnement.

13. Nous remercions notre collègue Donato Bergandi qui a accepté de rechercher et traduire des documents se référant à la situation en Italie.

14. Cette charte a été l'élément préparatoire nécessaire à la mise en place d'un concours national permettant de présenter un projet expérimental sur l'éducation environnementale.

15. Charte signée entre les deux ministères intitulée "Carta d'intenti tra il Ministero dell'Istruzione, dell'Università e della Ricerca e il Ministero dell'Ambiente e della Tutela del Territorio e del Mare in materia di "Scuola, Ambiente e Legalità" » (Roma, 29 luglio 2009) : www.minambiente.it/export/sites/default/archivio/documenti/

Scuola_Ambiente_e_Legalita.pdf

16. Ministero dell'Istruzione, dell'Università e della Ricerca e il Ministero dell'Ambiente e della Tutela del Territorio e del Mare (2009), Oggetto : Linee guida per l'educazione ambientale e allo sviluppo sostenible : www.minambiente.it/export/sites/default/archivio/notizie/ Linee_guida_ScuolaxAmbiente_e_Legalitx_aggiornato.pdf

17. Nous préférons employer ici le vocabulaire français.

18. Dont les circulaires et les programmes disciplinaires de Géographie et de Sciences de la Vie et de la Terre (SVT), les plus impliqués dans la mise en place de l'EDD.

19. Colloques et rapports nationaux fondateurs, rapports d'étapes, documents d'accompagnement des programmes précités, Stratégies Nationales de Développement Durable (SNDD), etc.

20. Ces deux auteurs sont respectivement Inspecteurs Généraux de SVT et d'Histoire-Géographie.

\section{RÉSUMÉS}

L'analyse des diverses réponses du secteur de l'éducation à la recommandation de l'ONU de promouvoir le développement durable amène à observer un gradient de positionnement. Si certains choix nationaux sont imprégnés d'une vision éconocentrée et néolibérale (RoyaumeUni), d'autres pays comme la Hollande font coexister à côté de cette prescription un programme apparenté à une Éducation à la Nature et à l'Environnement. En Italie, en Suisse et en Allemagne, l'éducation relève d'une juridiction décentralisée, ce qui a pour conséquence la cohabitation de plusieurs systèmes éducatifs qui privilégient parfois des approches très diverses de l'éducation à la soutenabilité (ESD). Enfin, à l'opposé de la politique du Royaume-Uni, se trouvent plusieurs pays latino-américains qui proposent des approches nationales plus critiques et distantes de la 
prescription onusienne. Dans une présentation plus diachronique, mais néanmoins succincte, les prescriptions successives de l'éducation nationale française sont placées dans ce gradient de positionnement. À travers l'exemple français, nous montrerons comment les finalités et les valeurs sous-jacentes à la politique d'ESD choisie cadrent les savoirs à transmettre aux élèves.

The analysis of various educational responses to the recommendation of the UN to promote sustainable development leads to observe different positions. If some national choices are marked by a neoliberal and econocentric vision (United Kingdom), other countries as Holland make coexist in quoted by this prescription a program being similar to Nature and Environment Education. In Italy, Switzerland and Germany, education is an area of provincial jurisdiction, which means that many educational systems coexist, sometimes favoring very different approaches to ESD. Finally, in contrast to the policy of ESD in the UK, there are several Latin American countries which propose more critical and distant national approaches regarding the UN prescription. In a more diachronic but nevertheless brief presentation, the successive requirements of the French national education are placed in this diversity of positions. Through the French example, we shall show how the purposes and the underlying values in the political choice of ESD frame the knowledge to be passed on to the pupils.

\section{INDEX}

Index géographique : Italie, Pologne, Allemagne, Suisse

\section{AUTEURS}

\section{YVES GIRAULT}

Yves Girault est professeur au Muséum national d'Histoire naturelle, directeur adjoint de l'Unité mixte de recherche Patrimoines locaux et membre du Centre de recherche en éducation et formation relatives à l'environnement et à l'écocitoyenneté à l'UQAM (Montréal). Ses travaux portent notamment sur l'étude de l'évolution des discours scientifiques concernant les rapports Hommes - natures - sociétés dans le champ particulier du musée (analyse des enjeux sociopolitiques, des représentations identitaires des acteurs, du mode d'énonciation et des modes de représentations muséographiques). Cette analyse du discours scientifique a comme finalité de comprendre le rôle du média exposition dans les relations entre sciences, cultures et sociétés.

\section{AURÉLIE ZWANG}

Aurélie Zwang est doctorante au Muséum national d'Histoire naturelle, au sein de l'Unité mixte de recherche Patrimoines locaux. Également professeure agrégée de Sciences de la Vie et de la Terre et engagée dans des pratiques d'éducation au développement durable, elle contribue à la formation continue en EDD dans l'Académie de Paris. Sa thèse, à la croisée des sciences de l'éducation et des sciences de l'information et de la communication, porte sur les expositions itinérantes légitimées par l'éducation nationale comme médias éducatifs pour l'EDD.

\section{AGNIESZKA JEZIORSKI}

Agnieszka Jeziorski est doctorante en sciences de l'éducation en cotutelle internationale à l'Université d'Aix Marseille et à l'Université Laval, membre du laboratoire ADEF et du Centre de recherche en éducation et formation relatives à l'environnement et à l'écocitoyenneté de l'UQAM. Elle est également attachée temporaire d'enseignement et de recherche à l'IUT de Digne 
- Université d'Aix-Marseille. Ses recherches portent sur l'analyse des représentations sociales et la didactique des questions économiques, sociales et environnementales « socialement vives ». 